\title{
Saúde mental no contexto universitário
}

\author{
${\text { Marcelo de } \text { Costa }^{1} \text {; Yanne Barros Moreira }}^{2}$
}

\section{Resumo}

Em um relatório publicado pela FONAPRACE em 2011, quase metade dos universitários queixou-se de algum sofrimento emocional vivenciado nos últimos doze meses. $\mathrm{O}$ índice de depressão e ansiedade nos alunos universitários é maior que o encontrado na população em geral, trazendo o indício que o ambiente acadêmico é um forte fator de risco para o desenvolvimento da depressão. A excessiva carga de trabalhos acadêmicos é apontada como um fator estressante para os alunos, mas fatores como dificuldades de ordem financeira, em relação à mudança de cidade e em relação a dificuldades interpessoais também contribuem para a fragilidade do estudante. Durante o período acadêmico, essas dificuldades podem afetar o desempenho cognitivo do aluno e suas relações interpessoais, mas em um âmbito mais amplo, podem afetar o futuro de sua carreira profissional. A universidade deve repensar iniciativas de apoio emocional aos alunos como forma de amenizar estes problemas durante sua vida acadêmica.

Palavras Chave: Depressão ; Saúde Mental ; Universidade;

A transição entre escola e vida universitária é, para grande parcela dos jovens, a conquista de sua própria independência, porém também pode ser visto como um período crítico de adaptação ao novo ambiente e a novas exigências. Mais da metade dos alunos relata dificuldades no primeiro ano de universidade (HERR, 1992), por ser um período que pode tornar mais evidentes problemas pessoais, acadêmicos e financeiros dos alunos, elevando assim, os níveis de stress e ansiedade dos estudantes (FERRAZ, PEREIRA, 2002). Em um relatório sobre o perfil socioeconômico e cultural das universidades brasileiras (FONAPRACE, 2011), foi analisada a relação entre dificuldades emocionais e o desempenho acadêmico dos estudantes: $43 \%$ dos estudantes relataram dificuldades de adaptação em relação à mudança de cidade, moradia e distância da família, 46\% relataram dificuldade em relacionamentos interpessoais ou sociais e $52 \%$ apontaram as dificuldades financeiras como um fator que influenciava diretamente o desempenho

\footnotetext{
${ }_{1}^{1}$ Aluno do Bacharelado de Design Gráfico da Universidade Federal do Paraná, marcelodecosta@gmail.com

${ }^{2}$ Aluna do Bacharelado de Design Gráfico da Universidade Federal do Paraná, yanne.b.moreira@gmail.com
} 
acadêmico. Entre outros fatores estressantes encontrados na mesma pesquisa, estão a excessiva carga de trabalho, apontada por $37 \%$ dos estudantes e a carga de trabalhos acadêmicos, destacado por $58 \%$ dos estudantes.

Dentre os principais fatores ambientais que predispõem um indivíduo à depressão, o stress e a ansiedade estão diretamente relacionados aos indivíduos com depressão (JOCA, PADOVAN, GUIMARÃES, 2003), pois, em cerca de 60\% dos casos, os episódios depressivos são precedidos pela ocorrência de fatores estressantes, principalmente de origem psicossocial. Sendo o stress e o esgotamento fatores de risco para a depressão e outras condições, o ambiente universitário com a carga pesada de trabalho e fatores não diretamente ligados à vida acadêmica (como moradia, condições financeiras e habilidades sociais) porém relacionados com as dificuldades acadêmicas, quase metade $(47,7 \%)$ dos alunos universitários relatou ter vivido uma crise emocional recente (FONAPRACE, op. cit.).

\section{Impacto da depressão nos universitários}

De acordo com a Organização Mundial da Saúde (2012), a depressão é um transtorno mental comum que se apresenta com humor deprimido, perda de interesse ou prazer, diminuição de energia, sentimentos de culpa ou baixa autoestima, perturbação do sono ou apetite e concentração pobre. Além disso, a depressão muitas vezes vem atrelada a sintomas de ansiedade. Estes problemas podem tornar-se crônicos ou recorrentes e levar a substancial deficiências na capacidade de um indivíduo de cuidar das suas responsabilidades diárias. Na pior das hipóteses, a depressão pode levar ao suicídio. Embora a característica mais típica dos estados depressivos seja a proeminência dos sentimentos de tristeza ou vazio, nem todos os pacientes relatam a sensação subjetiva de tristeza. Os relatos de maior incidência é sobre a perda da capacidade de experimentar prazer nas atividades em geral e a redução do interesse pelo ambiente. Frequentemente associa-se à sensação de fadiga ou perda de energia, caracterizada pela queixa de cansaço exagerado. A depressão está entre as doenças mais incapacitantes e, anualmente, ocorrem mais de 1 milhão de mortes por suicídio (OMS, op. cit.). No diagnóstico da depressão levam-se em conta: sintomas psíquicos, fisiológicos e evidências comportamentais (DEL PORTO, 1999). 
Os índices de ansiedade e depressão encontrados nos estudantes universitários são elevados, sendo, inclusive, muito superiores aos encontrados na população geral, com os alunos primeiros anos da universidade apresentando a maior concentração de sintomas depressivos e ansiosos (IBRAHIM et al., 2013).

É fundamental, frente a estas informações, questionar sobre as consequências que esta vulnerabilidade representa na performance do estudante. Eisenberg et al.(2009) desenvolveram um estudo para compreender a relação entre a saúde mental dos estudantes universitários e o desempenho acadêmico durante o curso. Para tal, foi distribuído um questionário sobre distúrbios do foro psíquico, feita pela internet, a uma amostra aleatória de 2.800 estudantes da Universidade de Michigan no ano de 2005, e posteriormente, no ano de 2007 foi distribuído um questionário follow-up à mesma amostra. A análise dos dados obtidos com a aplicação dos questionários revelou que a depressão era o distúrbio mental com maior prevalência. Cerca de 14\% dos alunos sofriam de sintomatologia depressiva, 3\% de sintomatologia ansiosa e 3\% de distúrbios alimentares. Ao relacionar os dados da pesquisa sobre a saúde mental dos estudante com os índices de rendimento acadêmico dos mesmo, os autores concluíram que a depressão influencia significativamente no baixo desempenho acadêmico e aumenta a probabilidade de abandono escolar, aparecendo frequentemente associada à ansiedade.

\section{Adaptação, distância da família e bem-estar psicológico}

De acordo com a pesquisa da FONAPRACE (op. cit.), 34,58\% dos estudantes não reside com a família. Junto com as experiências da faculdade, morar longe do núcleo familiar pode ser um processo solitário e, caso haja um problema de adaptação, causar a condição conhecida como homesickness. Para o DMS-IV (1999), homesickness se trata de um problema de adaptação que mistura a ansiedade e o humor depressivo, causada na universidade pela distância entre os estudantes e seu lar de origem. Residir em local distante do núcleo afetivo de origem, de fato, tem sido apontado como importante variável no processo de investigação da saúde mental do estudante universitário. Fioravanti et al. (2005) identificaram que um número significativo de universitários, oriundos de localidades diferentes da região de localização da instituição de ensino, consideraram-se estressados $(89,29 \%)$. 
Quanto ao desempenho cognitivo, as saudades de casa podem reduzir a capacidade de concentração nas atividades diárias e de memorização dos alunos, conduzindo a falhas cognitivas, ao decréscimo do desempenho acadêmico e da percepção de controle sobre as exigências acadêmicas (VAN TILBURG, 2005). Nos estudos de Archer et al. (1998), verificou-se que os estudantes universitários com saudades de casa apresentaram níveis maiores de ansiedade e depressão, pensamentos obsessivos e falhas a nível cognitivo. $\mathrm{Na}$ sua forma mais intensa, pode precipitar em ausência e abandono escolar, na medida em que os estudantes com saudades de casa apresentam três vezes mais probabilidade de abandonar os estudos (THURBER, WALTON, 2012).

\section{Habilidades sociais e interpessoais}

Além dos fatores característicos da vida universitária, outros elementos presentes mesmo antes da graduação podem ser fatores de risco para a saúde mental do estudante. A ausência de apoio emocional necessário e a dificuldade para tirar dúvidas por timidez são fatores que fragilizam o estudante e o tornam mais propício a desenvolver algum transtorno (FIORETTI et al., 2010). Em um estudo realizado por Edmondson et al (2007), sobre os problemas relativos à saúde mental e sua relação com as habilidades sociais, dados indicam que alunos que apresentaram maior nível de nervosismo e tristeza possuíam pontuação menor em competência social e expressividade afetiva.

De um outro lado, o estudante com depressão, ou indicação para o quadro depressivo, também apresenta dificuldades em relação a suas habilidades sociais devido a sua condição (BOLSONI-SILVA, GUERRA, 2014). Por consequência, esses universitários obtém poucos reforçadores nas interações sociais, o que justifica, de certa forma, o seu quadro de depressão.

\section{Estratégias}

Tendo em vista os problemas apresentados anteriormente, é de extrema importância que existam iniciativas da instituição para auxiliar os alunos e ampará-los. As intervenções psicológicas na universidade são importantes, não considerando somente o período de estudos, mas também as consequências que a depressão pode causar na vida do aluno após formado ao se inserir no mercado de trabalho, já que um profissional apresentaria baixa competência social e comunicação ineficaz (MURAKAMI et al., 2008). Esses 
problemas que podem se agravar e influenciar de forma negativa a economia e a produtividade dos profissionais formados pela universidade pode ser amenizada quando tratadas enquanto ainda se é um jovem adulto.

A instituições de ensino também devem ser responsáveis por informar e orientar os alunos quantos aos sintomas, benefícios da ajuda psicológica profissional e criar um diálogo que possa fazer com que os alunos se abram mais ao tratamento de problemas emocionais, já que a informação é um fator chave na busca e sucesso do tratamento (THOMAS et al., 2014). Nas intervenções também tornam-se importantes o treino das habilidades sociais como forma de ensinar diversas classes de respostas a serem realizadas em diversas interações como forma de gerar mais reforçadores para o estudante (BOLSONI-SILVA, GUERRA, op. cit.). Existe também a necessidade da existência de serviços especializados para estudantes que se encontram longe da família ou com dificuldades de adaptação às exigências acadêmicas. Para esses alunos recémchegados e, sobretudo para os que deixaram a sua residência de origem parece importante algumas iniciativas de acolhimento, que passam pelo conhecimento da cidade onde se situa a instituição e respectiva comunidade envolvente, assim como pela explicação quer do currículo do curso, quer do próprio regulamento acadêmico, fazendo deste modo com que o aluno aos poucos se "sinta em casa" (GONÇALVES, 2010).

\title{
Mental health in the university environment.
}

\author{
Abstract \\ In a report published by FONAPRACE in 2011, almost half of the university students \\ complaint about emotional struggles experienced in twelve months. The levels of depression \\ and anxiety in university students is higher than the levels found in the general population, \\ bringing to light that the academic environment is a strong risk factor for the development of \\ conditions like depression. The excessive amount of academic work is pointed as a key \\ stressing factor for students, but financial struggles, difficulties about moving cities and \\ difficulties in interpersonal relationships also contribute to their emotional fragility. During \\ their academic term, these difficulties can affect their cognitive performance and their
}


interpersonal relationships, but in a wider sense, it can also affect the future of their professional careers. The university must rethink initiatives of emotional support offered to students as a way of ease those issues during their academic life.

Keywords: Depression. Mental Health. University,

\section{Referências}

ARCHER, J. et al. "Derivation of a homesickness scale". British Journal of Psychology, v. 89 , n. 2, p. 205-221, maio 1998.

BOLSONI-SILVA, A. T.; GUERRA, B. T. "O impacto da depressão para as interações sociais de universitários”. Estudos e Pesquisas em Psicologia, v. 14, n. 2, p. 429-452, 10 set. 2014.

DEL PORTO, J. A. “Conceito e diagnóstico da depressão”. Rev. Bras. Psiquiatria, 21 (supl. Depressão), 6- 11, 1999.

DSM-IV. JAMA, v. 282, n. 4, p. 387, 28 jul. 1999.

EDMONDSON, C. B.; CONGER, J. C.; CONGER, A. J. "Social skills in college students with high trait anger." Journal of Social and Clinical Psychology, v. 26, n. 5, p. 575594, maio 2007.

EISENBERG, D.; GOLBERSTEIN, E.; HUNT, J. B. "Mental health and academic success in college". The B.E. Journal of Economic Analysis \& Policy, v. 9, n. 1, 15 jan. 2009.

FERRAZ, M. F., \& PEREIRA, A. S. "A dinâmica da personalidade e o homesickness (saudades de casa) dos jovens estudantes universitários”. Psicologia, Saúde \& Doenças, 2002, p. 149-164.

FIORAVANT, A. R., SHAIANI, D. A., BORGES, R. C., \& BALIERO, R. C. "Estudo sobre os fatores de stress entre alunos da Unicamp.” Revista Ciências do Ambiente On-line, p. 41-48, 2005.

FIOROTTI, K. P. et al. "Transtornos mentais comuns entre os estudantes do curso de medicina: Prevalência e fatores associados.” Jornal Brasileiro de Psiquiatria, v. 59, n. 1, p. 17-23, 2010.

FÓRUM NACIONAL DE PRÓ-REITORES DE ASSUNTOS COMUNITÁRIOS E ESTUDANTIS Perfil socioeconômico e cultural dos estudantes de graduação das instituições federais de ensino superior. Brasília: FONAPRACE, 2011

GONÇAlVES, L. M. C. Saudades de Casa, Depressão e Personalidade em Estudantes Universitários, 2010 
HERR, E. L., \& CRAMER, S. H. Carer guidance and counseling through the life span. Systematic approaches. Nova Iorque: Harpe Collins Publishers, 1992

IBRAHIM, A. K. et al. "A systematic review of studies of depression prevalence in university students.” Journal of Psychiatric Research, v. 47, n. 3, p. 391-400, mar. 2013.

JOCA, S. R. L.; PADOVAN, C. M.; GUIMARÃES, F. S. “Estresse, depressão e hipocampo”. Revista Brasileira de Psiquiatria, v. 25, p. 46-51, dez. 2003.

MURAKAMI, K. et al. "Learning on work placement: The narrative development of social competence.” Journal of Adult Development, v. 16, n. 1, p. 13-24, 21 out. 2008.

\section{ORGANIZAÇÃO MUNDIAL DA SAÚDE. DEPRESSION: A Global Public Health}

Concern Geneva : World Health Organization,2012. Disponível em:

<http://www.who.int/mental_health/management/depression/who_paper_depression_wf mh_2012.pdf> Acesso em: 31 abr. 2016.

THOMAS, S. J.; CAPUTI, P.; WILSON, C. J. Specific attitudes which predict psychology students' intentions to seek help for psychological distress. Journal of Clinical Psychology, v. 70, n. 3, p. 273-282, 1 jul. 2013.

THURBER, C. A.; WALTON, E. "Preventing and treating homesickness." Official Journal of the American Academy of Pediatrics, v. 119, n. 1, p. 192-201, 1 jan. 2007.

VAN TILBURG, M. Psychologiocal aspects of geographical moves: Homesickness and acculturation stress. 2a ed. Amsterdam: Amsterdam University Press., 2005. 